\title{
Optimal Control of the Coronavirus Pandemic with Impacts of Implemented Control Measures
}

\author{
T. T. Yusuf, A. Abidemi*, A. S. Afolabi, E. J. Dansu \\ Department of Mathematical Sciences, Federal University of Technology, Akure, Nigeria
}

\begin{abstract}
This paper considers the current global issue of containing the coronavirus pandemic as an optimal control problem. The goal is to determine the most advantageous levels of effectiveness of the various control and preventive measures that should be attained in order to effectively reduce the burden of the disease within a relatively short time. Thus, the problem's objective functional is constructed such that it minimizes the prevalence as well as the cost of implementing four control measures (namely personal protection, contact tracing and testing control for asymptotically infected individuals, detection control for symptomatic infected individuals and management control for hospitalized individuals) subject to a model for the disease transmission dynamics which incorporates four time-dependent control variables. The optimality system of the model is derived based on Pontryagin's maximum principle. Thereafter, the resulting optimality system is solved numerically using the Runge-Kutta fourth order scheme with forward-backward sweep approach to evaluate the effect of combining at least any three of the control functions with personal protection always in use on the disease dynamics. Findings from our results show that the new cases and the prevalence of the disease can be remarkably reduced in a cost effective way by implementing any of the control strategies analysed in this work. However, the results of efficiency analysis suggest that a strategy which combines all the four preventive and control measures is most effective in reducing the disease burden in the population.
\end{abstract}

\section{DOI: $10.46481 /$ jnsps.2022.414}

Keywords: Coronavirus, Epidemiological model, Objective functional, Optimality system, Pontryagin's maximum principle, Disease prevalence

Article History :

Received: 18 September 2021

Received in revised form: 13 December 2021

Accepted for publication: 06 February 2022

Published: 28 February 2022

(C)2022 Journal of the Nigerian Society of Physical Sciences. All rights reserved. Communicated by: T. Latunde

\section{Introduction}

The coronavirus disease (COVID-19), which originated from China towards the end of the year 2019, has the severe acute respiratory syndrome coronavirus-2 (SARS-CoV-2) as its causative agent. Just like similar infections caused by coronaviruses, namely the severe acute respiratory syndrome (SARS) and the middle east respiratory syndrome (MERS), the tendency of rapid

\footnotetext{
${ }^{*}$ Corresponding author tel. no: +2348036412201

Email address: aabidemi@futa.edu.ng (A. Abidemi )
}

spread of COVID-19 calls for concern [1]. Coronaviruses are said to have the most common genes of the ribonucleic acid viruses, though with possibility of genetic mutations.

COVID-19 is transmitted from humans to humans by inhaling the respiratory droplets from humans infected by COVID19 during coughing and sneezing and through direct contact with contaminated surfaces $[2,3]$. The incubation period for COVID-19 ranges between 0 and 14 days, although a longer incubation period of 25 days has also been reported in literature. The mortality rate of the disease is lower than the recovery rate, but this ratio varies from one country to another as well as re- 
gion to region. The primary symptoms of COVID-19 include body aches, headache, consistent dry coughing, severe chest pain, high fever, and complication in the respiratory system [3].

Presently, there are no certified potent drugs for the treatment of the disease. Though, there are ongoing efforts in this direction. Recently, Wu et al. undertook a review of therapies to prevent the contagion, control the spread and support patients infected with the disease through the use of antiviral, and supporting agents [4]. Also, Galluccio et al. proposed a multifaceted management approach in handling the complexity presented by the global COVID-19 pandemic [5]. They encourage knowledge sharing, proper timing and setup of treatments as possible ways out of the situation. In a related study, Becker investigated the usefulness of two cheap oral drugs -chloroquine and hydroxychloroquine- in the treatment of the disease based on the fact that these drugs had hitherto been helpful in managing autoimmune illnesses [6].

In another study conducted by Galvin et al., they found control and preventive interventions like the use of masks, sanitizers, social distancing and others to be quite helpful in curbing the spread of COVID-19 in Taiwan [7]. Moreover, the foregoing is equally true in other parts of the world since these measures limit contacts and the possibility of transmission among people as demonstrated in the studies by Shah et al. and Tsay et al. [8, 9]. Similarly, Ngonghala et al. developed a model to evaluate the impact of non-pharmaceutical approaches on COVID-19, namely social distancing, contact tracing, quarantine, isolation and the use of face mask [10]. These non pharmaceutical measures were found to be reliably effective based on the analysis of the model simulated using data from the United States of America. In addition, another closely related study with similar findings was conducted by Okuonghae and Omame, though their focus was on Lagos, which is the epicenter of COVID-19 in Nigeria [2].

Globally, there were $263,563,622$ confirmed cases of the coronavirus disease and 5,232,562 deaths due to the disease as at 3rd December 2021 [11]. Moreover, on the Nigerian scene, the first reported case of COVID-19 was on the 27th February 2020 due to an Italian visitor $[12,13,14]$. However, as of 5th December 2021, 3,580,510 COVID-19 samples had been tested in Nigeria, there had been 214,567 confirmed cases, 207,427 discharged cases, 2,980 cases of death; and 4,160 active cases [14]. According to the Nigeria Centre for Disease Control (NCDC) from 27th February, 2020 up to 31st July, 2020, all the thirty-six states of the Federal Republic of Nigeria and the Federal Capital Territory (FCT) had reported cases of COVID-19 [13]. Thus, it was instructive for Ohia [15] to express the need for urgent attention as it concerns contextualizing the peculiarity of Africa at large and Nigeria in particular as it relates to the management of COVID-19. This was necessary considering the deplorable state of the nation's health system and the dearth of infrastructure for emergency care for victims of the pandemic. However, having put up a successful fight against polio and Ebola in the past, Ebenso and Otu were of the opinion that Nigeria had some leverage in confronting this COVID-19 outbreak despite the below average capacity of the health system [16]. Nevertheless, Osseni opines that in spite of the fragile health care system in
sub-Saharan Africa, COVID-19 presents a huge opportunity for the region to demonstrate that they are equal to the task of combating the pandemic [17]. It is worthy of note that Nigeria is a major player in the region with a large human population, thus all eyes are on the country to see how it responds to this disease of global concern.

In recent years, optimal control theory has been applied to help inform policies and strategies which could be used in the control of infectious diseases. It is often used to determine the optimal levels of effectiveness of the different control measures that should be deplored per unit time in order to promptly contain an epidemic. For instance, Ijalana and Yusuf used the optimal control theory approach on a model describing the transmission dynamics of hepatitis B virus in highly endemic areas and recommended how the various control measures should be combined in order to effectively contain the disease in the area within a decade [18]. Similarly, Yusuf and Olayinka used the same approach to propose strategies for containing the meningitis disease recurrence in the meningitis belt of Africa [19]. In the same vein, Obsu and Balcha applied the theory on a model for COVID-19 spread and they found that the prevention of infection, intensive care for the hospitalized and sanitizing of infected sites are the most effective strategies against the disease's spread [20]. Equally, Yousefpour et al. worked on a model for COVID-19 with a multi-objective genetic algorithm for rates of contact and transition to quarantine. They came up with optimal policies for curtailing the disease while they also evaluated the economic impact of the disease [21].

Abioye et al. [22] emphasised the possibility of the eradication of COVID-19 in Nigeria if three control strategies, namely enforcement of preventive measures; prompt testing and treatment; and prevention of repeat infections, are duly sustained. Using the Prophet package, Abioye et al. [23] was able to predict a reliable trend in the evolution of COVID-19 infections in Nigeria for the period considered by the study. Diagne et al. [24] formulated and analyzed a COVID-19 transmission model which highlights the significance of effective contact rate, vaccine efficacy, vaccination rate, and proportion of symptomatic exposed individuals in the spread of the disease. Moreover, awareness programs and timely hospitalization were shown as very crucial in the containment of COVID-19 in Nigeria and other countries of the world by Musa et al. [25]. Also, Peter et al. [26] investigated the behaviour of COVID-19 infections based on fractional differentiation using the Atangana-Baleanu fractional derivative operator to establish existence. The iterative Laplace transform method was employed to carry out numerical simulations. The most important factors in the model were identified to be the force of infection and the progression rate of individuals from the exposed class to the infectious class. Using real data from Pakistan, Peter et al. [27] presented a novel model in order to understand the COVID-19 outbreak. The work highlighted that public health policies leading to properly managed transmission rates were necessary for effective management of the disease.

Most of the works on mathematical analysis of COVID-19 transmission dynamics do not consider the effect of combining most or all available control measures in an optimal way. That 
is, controlling the pandemic in a way that the prevalence, incidence and cost of implementing the adopted control strategies are minimised while the efficiency of the proposed strategies is quantitatively evaluated. The foregoing is critical to the implementation of any proposed control strategy, since it guarantees measurable achievement on fund invested. Consequently, this paper tries to fill this research gap as much as possible. In this work, we investigate optimal control strategies for containing the COVID-19 pandemic with a focus on Nigeria. The goal is to determine how to optimally combine the different disease control and preventive measures in order to contain the disease within the shortest possible time in a cost effective way. To this end, we formulate an optimal control problem involving a non-autonomous system of ordinary differential equations incorporating four time-dependent control variables representing preventive (precautionary) measures, contact tracing and testing control for asymptomatically infected individuals, detection control for symptomatic infected individuals, and management control for hospitalized individuals to describe the transmission dynamics of COVID-19 in a population. Pontryagin's maximum principle is used to conduct the analysis of the formulated optimal control problem to determine the necessary conditions for the optimal strategies for controlling the disease spread in the community. The paper outline is as follows: Section 2 describes the non-autonomous deterministic model for the spread of the disease; Section 3 contains the formulation of the optimal control problem, proof of existence of optimal control, its characterization, and the derivation of the optimality system; estimation of model parameters and initial conditions is considered in Section 4; Section 5 has the numerical simulations and efficiency analysis; conclusion is made in Section 6.

\section{Mathematical Model}

We consider an improved version of the COVID-19 transmission dynamics by Yusuf et al.[28]. This work is different from the former because it considers a non-autonomous deterministic model for COVID-19 transmission dynamics with time-dependent control measures. In addition, the goal of this paper is to solve an optimal control problem subject to the proposed model dynamics. Thus, the results will not just be about the state variables, rather it will include the adjoint and the control variables. So, the solution determines optimal level of each of the control variables that should be applied at any point in time in order to minimize the incidence and prevalence of the disease as well as the cost of implementing these control and preventive measures. Conventionally, the model subdivides the total population under consideration, denoted as $N(t)$, into five mutually exclusive compartments, namely: Susceptible $S(t)$, Asymptomatically infected $E(t)$, Symptomatically Infected $I(t)$, Hospitalized $H(t)$, and Recovered $R(t)$ compartments. Thus, $N(t)=S(t)+E(t)+I(t)+H(t)+R(t)$. The susceptible compartment contains individuals who can be infected with COVID-19 disease if sufficiently exposed to the infection without taking adequate protective measures; the asymptomatically infected compartment is made up of individuals who have been infected with the disease, though do not exhibit the symptoms of the infection but can infect others; the infected compartment is made up individuals who have been infected and show symptoms of the infection; the hospitalized compartment comprises of individuals who have been confirmed to be infected with disease based on standard tests and are placed under isolation in designated isolation centres; the recovered compartments contains individuals who have recovered from the infection and now possess temporary immunity against re-infection.

Furthermore, four time-dependent control functions, $u_{i}(t)$ $(i=1, \ldots, 4)$, are incorporated into the model to mitigate the spread of the disease in the population, where $u_{1}(t)$ represents the fraction of the whole population that adopt human personal protective intervention such as wearing of face mask, regular hand washing cum sanitizing and observing social distancing, $u_{2}(t)$ accounts for the successful effort of the contact tracing and testing for asymptomatically infected individuals, $u_{3}(t)$ denotes the successful detection effort for symptomatic infectious individuals, and lastly, the medical care successful effort (management control) for hospitalized individuals in designated isolation centers is represented by $u_{4}(t)$.

Therefore, the non-autonomous model system of ordinary differential equations governing the disease spread is as given in (1) as

$$
\begin{aligned}
\frac{d S}{d t}= & \Lambda-\left(1-u_{1}(t)\right)\left(\beta_{1} E+\beta_{2} H+\beta_{3} I\right) S-\mu S \\
& +\omega R, \\
\frac{d E}{d t}= & \left(1-u_{1}(t)\right)\left(\beta_{1} E+\beta_{2} H+\beta_{3} I\right) S-u_{2}(t) E \\
& -\rho E-\mu E, \\
\frac{d I}{d t}= & \rho E-u_{3}(t) I-\gamma I-\left(\mu+\delta_{2}\right) I, \\
\frac{d H}{d t}= & u_{2}(t) E+u_{3}(t) I-u_{4}(t) H-\left(\mu+\delta_{1}\right) H, \\
\frac{d R}{d t}= & \gamma I+u_{4}(t) H-\mu R-\omega R,
\end{aligned}
$$

with given initial conditions at time $t=0$ while the model parameters are as defined in Table 1.

Note that $\lim _{t \rightarrow \infty} N(t) \leq \frac{\Lambda}{\mu}$. However, under the dynamics described by (1), the region $\Omega$ defined by

$$
\Omega=\left\{(S, E, I, H, R) \in \mathbb{R}_{+}^{5} \mid S+E+I+H+R \leq \frac{\Lambda}{\mu}\right\}
$$

is positively invariant.

\section{Optimal Control Problem Formulation}

In this work, the objective functional is designed such that it is quadratic in control terms to follow the standard in literature $[18,19,29,30,31,32,33]$. Thus, our objective functional is defined as

$$
\begin{aligned}
\mathbb{Z}= & \min _{u_{1}, u_{2}, u_{3}, u_{4}} \int_{0}^{t_{f}}\left(w_{1} E+w_{2} I+w_{3} H\right. \\
& \left.+\frac{w_{4}}{2} u_{1}^{2}+\frac{w_{5}}{2} u_{2}^{2}+\frac{w_{6}}{2} u_{3}^{2}+\frac{w_{7}}{2} u_{4}^{2}\right) d t
\end{aligned}
$$


Table 1: Description of model parameters

\begin{tabular}{ll}
\hline \hline Parameter & Description \\
\hline$\Lambda$ & recruitment rate into the $S$ class \\
$\mu$ & natural death rate \\
$\delta_{1}$ & COVID-19 induced death rate for hospitalized individuals \\
$\delta_{2}$ & COVID-19 induced death rate for symptomatically infected individuals not hospitalized \\
$\beta_{1}$ & transmission rate of the disease based on unprotected contact with individuals in the $E$ class \\
$\beta_{2}$ & transmission rate of the disease based on unprotected contact with individuals in the $H$ \\
$\beta_{3}$ & class \\
$\rho$ & transmission rate of the disease based on unprotected contact with individuals in the $I$ class \\
$\gamma$ & progression rate from the $E$ class into the $I$ class \\
$\omega$ & recovery rate of individuals in the $I$ class \\
\hline \hline
\end{tabular}

subject to the dynamical system (1) with appropriate states initial conditions and $t_{f}$ is the final time while the control set $\mathbb{U}$ is Lebesgue measurable and it is defined as

$$
\begin{aligned}
\mathbb{U}= & \left\{\left(u_{1}(t), u_{2}(t), u_{3}(t), u_{4}(t)\right) \mid 0 \leq u_{i} \leq u_{i \max }<1,\right. \\
& \left.i=1, \ldots, 4, t \in\left[0, t_{f}\right]\right\} .
\end{aligned}
$$

In addition, the weight constants $w_{i}>0(i=1,2, \ldots, 7)$ that appear in (2) are the relative weights, and they ensure that each term in the integrand does not dominate while also balancing terms therein. Nevertheless, it is important to note that $w_{4}, w_{5}$, $w_{6}, w_{7}$ are relative measures of the cost or effort required to implement each of the associated controls while $w_{1}, w_{2}, w_{3}$ are relative measures of the importance of reducing the associated classes on the spread of COVID-19 in the country under consideration.

The upper bounds $\left(u_{1 \max }, u_{2 \max }, u_{3 \max }, u_{4 \max }\right)$ for each of the controls will depend on the budget allocated for the execution of each of the control measures and the practicability of achieving the set bounds. For this study, we shall hypothetically set $u_{1 \max }=0.7, u_{2 \max }=0.5, u_{3 \max }=0.5$, and $u_{4 \max }=0.9$ in our simulations with the assumptions that it is reasonably practicable to achieve the set bounds subject to the availability of needed resources. We wish to determine the optimal combination of different levels of effectiveness of the controls $u_{1}, u_{2}, u_{3}$ and $u_{4}$ that will be adequate to minimize the cost of implementing the preventive measures, the cost of contact tracing of the exposed individuals, the cost of medical testing of infected individuals, and the cost associated with hospitalizing cum treating hospitalized individuals as well as reducing the prevalence of the disease within a set time frame.

\subsection{Existence of an Optimal Control Strategy for the Problem}

Now, we examine the sufficient conditions for the existence of a solution to the optimal control problem.

Theorem 3.1. There exists an optimal control set $\left(u_{1}^{*}, u_{2}^{*}, u_{3}^{*}, u_{4}^{*}\right)$ with corresponding solution

$\left(S^{*}, E^{*}, I^{*}, H^{*}, R^{*}\right)$ to the model system (1) that minimize $\mathbb{Z}$ over U.
Proof. The existence of the optimal control is guaranteed by the compactness of the control and the state space and the convexity in the problem based on Theorem 3.1 and its corresponding Corollary in Fleming and Rishel[34]. The following non-trivial requirements from Fleming and Rishel's theorem are stated and verified below:

(1) The set of all solutions to the model equations (1) and its associated initial conditions together with the corresponding control functions in $\mathbb{U}$ is non empty.

(2) The state system can be written as a linear function of the control variables with coefficients dependent on time and state variables.

(3) The integrand $L$ in (2) is convex on $\mathbb{U}$ and additionally satisfies

$$
\begin{gathered}
L\left(t, S, E, I, H, R, u_{1}, u_{2}, u_{3}, u_{4}\right) \\
\quad \geq c_{1}\left\|\left(u_{1}, u_{2}, u_{3}, u_{4}\right)\right\|^{\alpha}-c_{2},
\end{gathered}
$$

where $c_{1}, c_{2}>0$ and $\alpha>1$.

We refer to Theorem 3.1 by Picard-Lindelof in [35]. Based on this theorem, if the solutions to the state equations are a priori bounded and if the state equations are continuous and Lipschitz in the state variables, then there is a unique solution corresponding to every admissible control set in $\mathbb{U}$. Using the fact that for all $(S, E, I, H, R) \in \Omega$, all the model states are bounded below and above, then the solutions to the state equations are bounded. In addition, it is direct to show the boundedness of the partial derivatives with respect to the state variables in the system and this shows that the system is Lipschitz with respect to the state variables. Hence, the proof of the first condition is established.

Equally, direct observation of the state equations (1) shows that they are linearly dependent on the controls $u_{1}, u_{2}, u_{3}$ and $u_{4}$. Thus, the second condition is also satisfied.

In order to establish the third condition, it is observed that the integrand $L$ in our objective functional is convex since it is quadratic in the controls. Thus, it suffices to show that there exists a bound on $L$ to satisfy the remaining condition. This is 
shown as below:

$$
\begin{aligned}
L= & w_{1} E+w_{2} I+w_{3} H+\frac{1}{2}\left(w_{4} u_{1}^{2}+w_{5} u_{2}^{2}\right. \\
& \left.+w_{6} u_{3}^{2}+w_{7} u_{4}^{2}\right) \\
\geq & \frac{1}{2}\left(w_{4} u_{1}^{2}+w_{5} u_{2}^{2}+w_{6} u_{3}^{2}+w_{7} u_{4}^{2}\right) \\
& \quad \text { ince } w_{i}>0, i=1, \ldots, 7 \\
\geq & \frac{1}{2}\left(w_{4} u_{1}^{2}+w_{5} u_{2}^{2}+w_{6} u_{3}^{2}+w_{7} u_{4}^{2}\right)-w_{4} \\
& \quad \operatorname{since} 0 \leq u_{1}<1, w_{4} u_{1}^{2}-w_{4} \leq 0 \\
\geq & \min \left(\frac{1}{2} w_{4}, \frac{1}{2} w_{5}, \frac{1}{2} w_{6}, \frac{1}{2} w_{7}\right)\left(u_{1}^{2}+u_{2}^{2}+u_{3}^{2}+u_{4}^{2}\right) \\
& -w_{4} \\
\geq & K\left\|\left(u_{1}, u_{2}, u_{3}, u_{4}\right)\right\|^{2}-w_{4} \\
& \quad \text { where } K=\min \left(\frac{1}{2} w_{1}, \frac{1}{2} w_{2}, \frac{1}{2} w_{3}, \frac{1}{2} w_{4}\right) .
\end{aligned}
$$

The foregoing shows that the integrand $L$ is bounded below. Hence, there exists a unique solution to the optimality system for small time intervals based on the opposite time orientations of the state and adjoint equations. In addition, the uniqueness of the solution of the optimality system guarantees the uniqueness of the optimal control if it exists.

\subsection{Characterization of the Optimal Controls}

We proceed to characterize the optimal controls $u_{1}^{*}, u_{2}^{*}, u_{3}^{*}, u_{4}^{*}$ which gives the optimal levels for the various control measures and the corresponding states $\left(S^{*}, E^{*}, I^{*}, H^{*}, R^{*}\right)$. The necessary conditions for the optimal controls are obtained using the optimality conditions of the Pontryagin's maximum principle [36] while the terminal conditions on the adjoint variables are obtained based on its transversality conditions [37].

\section{Theorem 3.2. (Necessary conditions) Let}

$\left(u_{1}^{*}, u_{2}^{*}, u_{3}^{*}, u_{4}^{*}\right) \in \mathbb{U}$ be an optimal control with the corresponding states $S^{*}, E^{*}, I^{*}, H^{*}$ and $R^{*}$. Then, there exist the adjoint variables $\lambda_{i}$ for $i=1, \ldots, 5$ which satisfy

$$
\begin{aligned}
\frac{d \lambda_{1}}{d t}= & \lambda_{1} \mu+\left(\lambda_{1}-\lambda_{2}\right)\left(1-u_{1}\right)\left(\beta_{1} E+\beta_{2} H+\beta_{3} I\right), \\
\frac{d \lambda_{2}}{d t}= & -w_{1}+\lambda_{2} \mu+\left(\lambda_{1}-\lambda_{2}\right)\left(1-u_{1}\right) \beta_{1} S \\
& +\left(\lambda_{2}-\lambda_{3}\right) \rho+\left(\lambda_{2}-\lambda_{4}\right) u_{2}, \\
\frac{d \lambda_{3}}{d t}= & -w_{2}+\left(\lambda_{1}-\lambda_{2}\right)\left(1-u_{1}\right) \beta_{3} S+\left(\lambda_{3}-\lambda_{4}\right) u_{3} \\
& +\left(\lambda_{3}-\lambda_{5}\right) \gamma+\lambda_{3}\left(\mu+\delta_{2}\right), \\
\frac{d \lambda_{4}}{d t}= & -w_{3}+\left(\lambda_{1}-\lambda_{2}\right)\left(1-u_{1}\right) \beta_{2} S+\left(\lambda_{4}-\lambda_{5}\right) u_{4} \\
& +\lambda_{4}\left(\mu+\delta_{1}\right), \\
\frac{d \lambda_{5}}{d t}= & \lambda_{5} \mu+\left(\lambda_{5}-\lambda_{1}\right) \omega
\end{aligned}
$$

and the transversality conditions

$$
\lambda_{i}\left(t_{f}\right)=0, \text { for } i=1, \ldots, 5
$$

with the optimal controls defined as follows

$$
\begin{aligned}
u_{1}^{*}= & \min \left\{\max \left(0, \frac{S\left(\beta_{1} E+\beta_{2} H+\beta_{3} I\right)\left(\lambda_{2}-\lambda_{1}\right)}{w_{4}}\right),\right. \\
& \left.u_{1 \max }\right\}, \\
u_{2}^{*}= & \min \left\{\max \left(0, \frac{E\left(\lambda_{2}-\lambda_{4}\right)}{w_{5}}\right), u_{2 \max }\right\}, \\
u_{3}^{*}= & \min \left\{\max \left(0, \frac{I\left(\lambda_{3}-\lambda_{4}\right)}{w_{6}}\right), u_{3 \max }\right\}, \\
u_{4}^{*}= & \min \left\{\max \left(0, \frac{H\left(\lambda_{4}-\lambda_{5}\right)}{w_{7}}\right), u_{4 \max }\right\} .
\end{aligned}
$$

Proof. Using Pontryagin's maximum principle, we obtain (5) from

$$
\begin{aligned}
& \frac{d \lambda_{1}}{d t}=-\frac{\partial \mathbb{H}}{\partial S}, \frac{d \lambda_{2}}{d t}=-\frac{\partial \mathbb{H}}{\partial E}, \frac{d \lambda_{3}}{d t}=-\frac{\partial \mathbb{H}}{\partial I}, \\
& \frac{d \lambda_{4}}{d t}=-\frac{\partial \mathbb{H}}{\partial H}, \frac{d \lambda_{5}}{d t}=-\frac{\partial \mathbb{H}}{\partial R},
\end{aligned}
$$

where the Hamiltonian $\mathbb{H}$ is given by

$$
\begin{aligned}
\mathbb{H}= & w_{1} E+w_{2} I+w_{3} H+\frac{w_{4}}{2} u_{1}^{2}+\frac{w_{5}}{2} u_{2}^{2} \\
& +\frac{w_{6}}{2} u_{3}^{2}+\frac{w_{7}}{2} u_{4}^{2} \\
+ & \lambda_{1}\left(\Lambda-\left(1-u_{1}\right)\left(\beta_{1} E+\beta_{2} H+\beta_{3} I\right) S\right. \\
& -\mu S+\omega R) \\
+ & \lambda_{2}\left(\left(1-u_{1}\right)\left(\beta_{1} E+\beta_{2} H+\beta_{3} I\right) S\right. \\
& \left.-u_{2} E-\rho E-\mu E\right) \\
+ & \lambda_{3}\left(\rho E-u_{3} I-\gamma I-\left(\mu+\delta_{2}\right) I\right) \\
+ & \lambda_{4}\left(u_{2} E+u_{3} I-u_{4} H-\left(\mu+\delta_{1}\right) Q\right) \\
+ & \lambda_{5}\left(\gamma I+u_{4} H-\mu R-\omega R\right) .
\end{aligned}
$$

The transversality conditions have the form (6), since all the states are free at the terminal time. Also, the Hamiltonian is maximized with respect to the controls at the optimal control $u^{*}=\left(u_{1}^{*}, u_{2}^{*}, u_{3}^{*}, u_{4}^{*}\right)$, thus we differentiate $\mathbb{H}$ with respect to $u_{1}$, $u_{2}, u_{3}$, and $u_{4}$ on $\mathbb{U}$ respectively to obtain

$$
\begin{gathered}
\frac{\partial \mathbb{H}}{\partial u_{1}}=w_{4} u_{1}-\left(\lambda_{2}-\lambda_{1}\right)\left(\beta_{1} E+\beta_{2} H+\beta_{3} I\right) S=0 \\
\quad \text { at } u_{1}=u_{1}^{*}, \\
\frac{\partial \mathbb{H}}{\partial u_{2}}=w_{5} u_{2}-\left(\lambda_{2}-\lambda_{4}\right) E=0 \text { at } u_{2}=u_{2}^{*}, \\
\frac{\partial \mathbb{H}}{\partial u_{3}}=w_{6} u_{3}-\left(\lambda_{3}-\lambda_{4}\right) I=0 \text { at } u_{3}=u_{3}^{*}, \\
\frac{\partial \mathbb{H}}{\partial u_{4}}=w_{7} u_{4}-\left(\lambda_{4}-\lambda_{5}\right) H=0 \text { at } u_{4}=u_{4}^{*} .
\end{gathered}
$$

Hence, solving for $u_{1}^{*}, u_{2}^{*}, u_{3}^{*}$, and $u_{4}^{*}$ on the interior of $\mathbb{U}$ 
gives

$$
\begin{aligned}
& u_{1}^{*}=\frac{S\left(\beta_{1} E+\beta_{2} H+\beta_{3} I\right)\left(\lambda_{2}-\lambda_{1}\right)}{w_{4}}, \\
& u_{2}^{*}=\frac{E\left(\lambda_{2}-\lambda_{4}\right)}{w_{5}}, \\
& u_{3}^{*}=\frac{I\left(\lambda_{3}-\lambda_{4}\right)}{w_{6}}, \\
& u_{4}^{*}=\frac{H\left(\lambda_{4}-\lambda_{5}\right)}{w_{7}} .
\end{aligned}
$$

We can now impose the bounds $0 \leq u_{1} \leq u_{1 \max }, 0 \leq u_{2} \leq$ $u_{1 \max }, 0 \leq u_{3} \leq u_{3 \max }$, and $0 \leq u_{4} \leq u_{4 \max }$ on the controls to get

$$
\begin{aligned}
u_{1}^{*}= & \min \left\{\max \left(0, \frac{S\left(\beta_{1} E+\beta_{2} H+\beta_{2} I\right)\left(\lambda_{2}-\lambda_{1}\right)}{w_{4}}\right),\right. \\
& \left.u_{1 \max }\right\}, \\
u_{2}^{*}= & \min \left\{\max \left(0, \frac{E\left(\lambda_{2}-\lambda_{4}\right)}{w_{5}}\right), u_{2 \max }\right\}, \\
u_{3}^{*}= & \min \left\{\max \left(0, \frac{I\left(\lambda_{3}-\lambda_{4}\right)}{w_{6}}\right), u_{3 \max }\right\}, \\
u_{4}^{*}= & \min \left\{\max \left(0, \frac{H\left(\lambda_{4}-\lambda_{5}\right)}{w_{7}}\right), u_{4 \max }\right\} .
\end{aligned}
$$

This ends the proof.

\section{Estimation of the Model Parameters and Initial Condi- tions}

\subsection{Estimation of Parameters}

The average new recruitment per unit time $(\Lambda)$ into the susceptible population was estimated using the annual increase in Nigeria's population from year 2000-2015. Nigeria's average annual population increase is estimated to be 3.922 million per year [38]. Thus, the daily new recruitment will be $\Lambda=\frac{3.922}{365}=0.0107$ humans per day.

In order to estimate the daily death rate of Nigerians, we used the average life expectancy of Nigerians which is estimated as $\frac{1}{2}(53+56)=54.5$ years and the concept that an individual losses the reciprocal of its life expectancy every day. This implies that $\mu=\frac{1}{365 \times 54.5}=0.00005$ per day [39].

Using the half-life concept, we estimate the disease progression rate $(\rho)$ into the symptomatic infected compartment based on the fact that about a fifth of asymptomatic infected individuals become symptomatic infected while it takes a maximum of 14 days for this transition to take place. So, $\rho=-\frac{1}{14} \ln (0.2)=$ 0.155 per day [40].

We estimated the natural recovery rate $(\gamma)$ based on the fact that about $80 \%$ of infected individuals recover without treatment while an average of about a quarter infected individuals recover on daily basis $[40,41]$. Thus, $\gamma=0.8 \times 0.25=0.2$ per day.

The disease-induced death rate $\left(\delta_{1}\right)$ is estimated from the Nigeria COVID-19 epidemiological data of 79 consecutive days [40]. So, we estimate it as $\delta_{1}=\frac{1}{79} \times \ln \frac{506}{2}=0.07$ per day. In addition, we assumed that those COVID-19 infected individuals who were not hospitalized are twice likely to die due to the infection. Hence, $\delta_{2}=2 \times 0.07=0.14$ per day.

Similarly, the disease transmission rate due to the exposed individuals $\left(\beta_{1}\right)$ is estimated using the Nigeria COVID-19 prevalence epidemiological data of 79 consecutive days [40]. So, we estimated $6 \beta_{1}=\frac{1}{79} \times \ln \frac{19808}{190}=0.0588$. Thus, $\beta_{1}=0.0098$ per day. Also, we assume that the hospitalized infected individuals are twice likely to transmit the disease when compared to asymptomatic infected individuals while the undetected symptomatically infected individuals are three times likely to transmit the disease when compared to asymptomatic infected individuals. Hence, we estimate $\beta_{2}=2 \times \beta_{1}=0.0196$ per day and $\beta_{3}=3 \times \beta_{1}=0.0294$ per day.

The disease waning rate $\omega$ is taken conservatively to be 0.005 per day, since the outbreak is unprecedented in human history. It is worth mentioning that this term is incorporated based on recent reports of cases of re-infection by the disease after recovery in some countries. We assumed that it will take about six months before an earlier infected person could be reinfected.

\subsection{Estimation of Initial Conditions}

We adopted the Nigerian coronavirus surveillance data for the 17th May, 2020. We assumed the total number of people tested for the virus as at the date as initial cohort for the disease spread through which the entire country might eventually become susceptible. So, we take the initial conditions for each of the compartments as $S(0)=28193, E(0)=1394, I(0)=4183$, $H(0)=4183, R(0)=1594$ with model parameter values as estimated in the preceding section.

\section{Numerical Simulations and Efficiency Analysis}

\subsection{Numerical Simulations}

The resulting optimality system consisting the dynamical system (1) and its associated initial conditions, the adjoint system (5), and the transversality conditions (6) coupled with control characterizations given in (7) is solved numerically using a fourth order iterative Runge-Kutta scheme implemented in MATLAB. This method solves the state equation with an initial guess for $u_{1}, u_{2}, u_{3}$ and $u_{4}$ forward in time; after which it solves the adjoint equations backward in time while the controls are continuously updated based on the control characterization in (7). The computational procedure is done iteratively until the results converge. See Lenhart and Workman[42] for details.

In order to investigate the impact of combined efforts of the controls $u_{i}(i=1, \ldots, 4)$ on the dynamics of COVID-19 pandemic in the population, the weight constants $w_{i}(i=1,2, \ldots, 7)$ that appear in the objective functional expressed by (2) are taken as $w_{i}=10$ with the assumption that the available resources can only accommodate the maximum levels of the controls as defined by $u_{1 \text { max }}=0.7, u_{2 \max }=u_{3 \max }=0.5$ and $u_{4 \max }=0.9$. Keeping in mind that precautionary measures such as wearing of face masks, observing social distancing when in public, regular personal hygiene (hand washing and sanitizing) play crucial roles in the fight against COVID-19 right from the onset of 
the pandemic outbreak, the effect of different combinations of personal protection (precautionary) measure with at least any two other control interventions on the transmission dynamics of COVID-19 in the community are investigated. Thus, four different control strategies are considered, and are defined as follows: Strategy 1: optimal use of personal protection, contact tracing and testing control for asymptomatically infected individuals, and detection control for symptomatic infected individuals $\left(u_{1}(t) \neq 0, u_{2}(t) \neq 0, u_{3}(t) \neq 0, u_{4}(t)=0\right)$, Strategy 2: optimal use of personal protection, contact tracing and testing control for asymptomatically infected individuals, and management control for hospitalized individuals $\left(u_{1}(t) \neq 0\right.$, $\left.u_{2}(t) \neq 0, u_{4}(t) \neq 0, u_{3}(t)=0\right)$, Strategy 3: optimal use of personal protection, detection control for symptomatic infected individuals, and management control for hospitalized individuals $\left(u_{1}(t) \neq 0, u_{3}(t) \neq 0, u_{4}(t) \neq 0, u_{2}(t)=0\right)$ and Strategy 4: optimal use of personal protection, contact tracing and testing control for asymptomatically infected individuals, detection control for symptomatic infected individuals, and management control for hospitalized individuals $\left(u_{1}(t) \neq 0, u_{2}(t) \neq 0\right.$, $\left.u_{3}(t) \neq 0, u_{4}(t) \neq 0\right)$.

\subsubsection{Strategy 1-Optimal Use of Personal Protection, Con- tact Tracing and Testing Control for Asymptomatically Infected Individuals, and Detection Control for Symp- tomatic Infected Individuals $\left(u_{1}(t) \neq 0, u_{2}(t) \neq 0, u_{3}(t) \neq\right.$ $\left.0, u_{4}(t)=0\right)$}

The effect of the control intervention that considers the simultaneous use of optimal personal protection $\left(u_{1}(t)\right)$, optimal contact tracing and testing control for asymptomatic infected individuals $\left(u_{2}(t)\right)$ and optimal detection control for symptomatic infectious humans $\left(u_{4}(t)\right)$ on the community spread of COVID19 is evaluated as shown in Figure 1. It is seen in Figure 1a that the size of COVID-19 prevalence decreases more rapidly with control intervention than when no control is applied in the population. Also, with application of this control strategy, the number of new cases is constantly managed near the initial number at the onset of the disease outbreak after about 50 days (counting from the commencement of the intervention) till the final time of the intervention (as shown in Figure 1b). Figure 1c reveals that personal protection $\left(u_{1}\right)$ is at maximum for about 190 days before undergoing stepwise fluctuation between the lower and upper bounds in the last intervention period, contact tracing and testing control for asymptomatic infected individuals $\left(u_{2}(t)\right)$ is held consistently close to the lower bound over the simulation period, while the detection control for symptomatic infectious humans $\left(u_{3}(t)\right)$ is at minimum level for about 175 days (counting from the beginning of the intervention period) before increasing and decreasing stepwisely between the lower and upper bounds in the remaining intervention period.

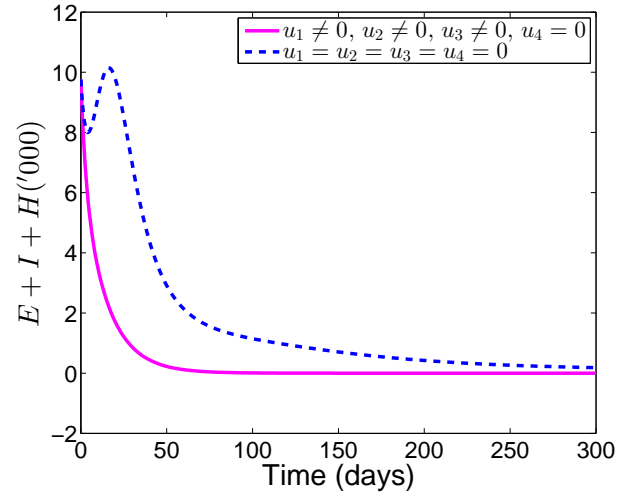

(a) Disease prevalence with and without control Strategy 1

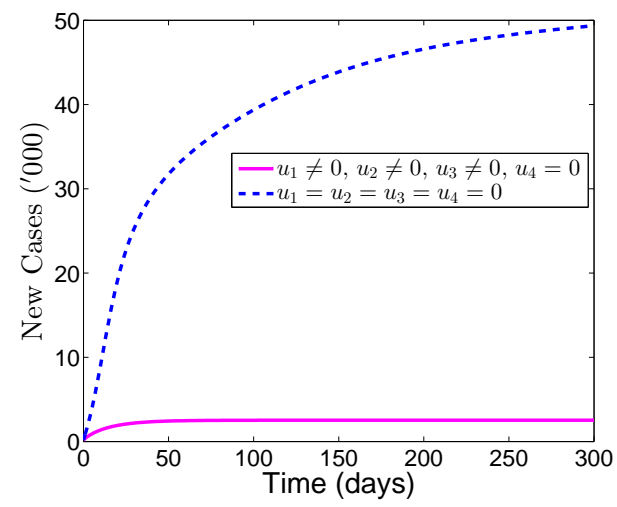

(b) COVID-19 incidence with and without Strategy 1

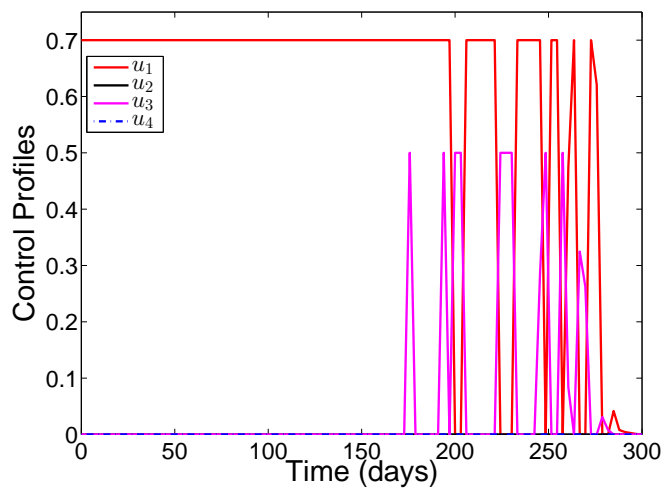

(c) Profiles of optimal controls $u_{1}, u_{2}, u_{3}$ with $u_{4}=0$

Figure 1: Simulations depicting the optimal use $u_{1}, u_{2}$ and $u_{3}$ only

\subsubsection{Strategy 2-Optimal Use of Personal Protection, Con-} tact Tracing and Testing Control for Asymptomatically Infected Individuals, and Management Control for Hospitalized Individuals $\left(u_{1}(t) \neq 0, u_{2}(t) \neq 0, u_{4}(t) \neq 0\right.$, $\left.u_{3}(t)=0\right)$

Figure 2 illustrates how the implementation of the control intervention that combines optimal use of personal protection $\left(u_{1}(t)\right)$, contact tracing and testing control for asymptomatic infected individuals $\left(u_{2}(t)\right)$ and management control for hospitalized individuals $\left(u_{4}(t)\right)$ affects the dynamics of transmission 


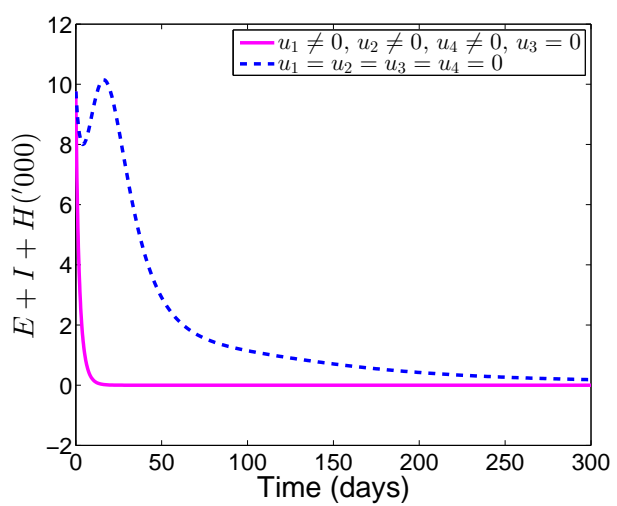

(a) Disease prevalence with and without control Strategy 2

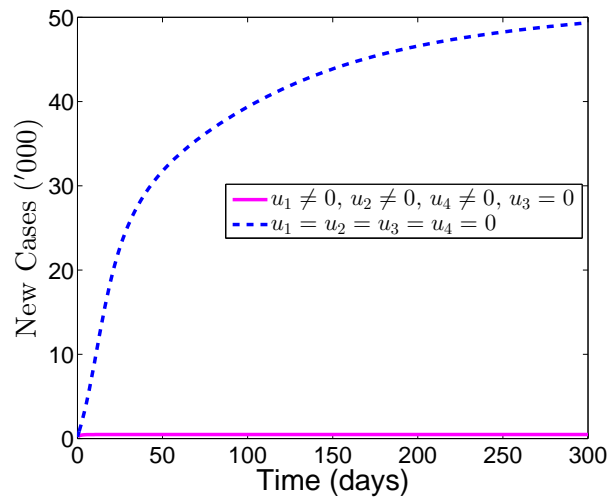

(b) COVID-19 incidence with and without Strategy 2

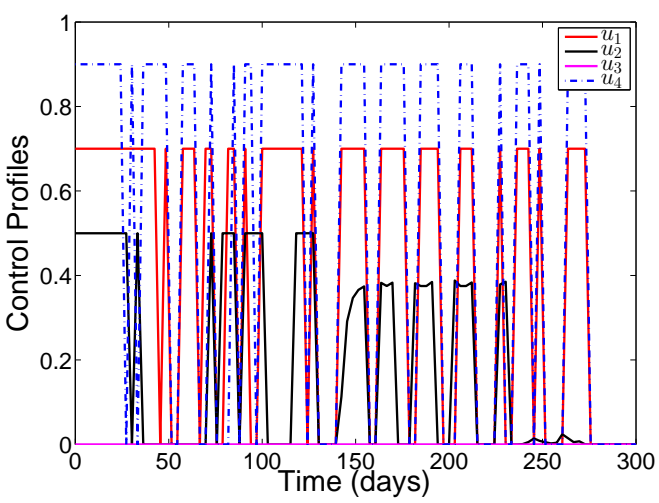

(c) Profiles of optimal controls $u_{1}, u_{2}, u_{4}$ with $u_{3}=0$

Figure 2: Simulations depicting the optimal use of $u_{1}, u_{2}$ and $u_{4}$ only

and spread of COVID-19 in the population. It is salient to state that with the implementation of this control strategy, the disease prevalence is sharply decreased to zero within a very short period as shown in Figure 2a. Further, the use of this intervention strategy helps to constantly hold the number of new cases at the initial value over the simulation time (see Figure 2b). All the three optimal controls $\left(u_{1}, u_{2}\right.$ and $\left.u_{4}\right)$ fluctuate between the maximum and minimum levels in a stepwise manner over the simulation period as revealed by the control profiles in Figure 2c.
5.1.3. Strategy 3-Optimal Use of Personal Protection, Detection Control for Symptomatic Infected Individuals, and Management Control for Hospitalized Individuals $\left(u_{1}(t) \neq\right.$ $\left.0, u_{3}(t) \neq 0, u_{4}(t) \neq 0, u_{2}(t)=0\right)$

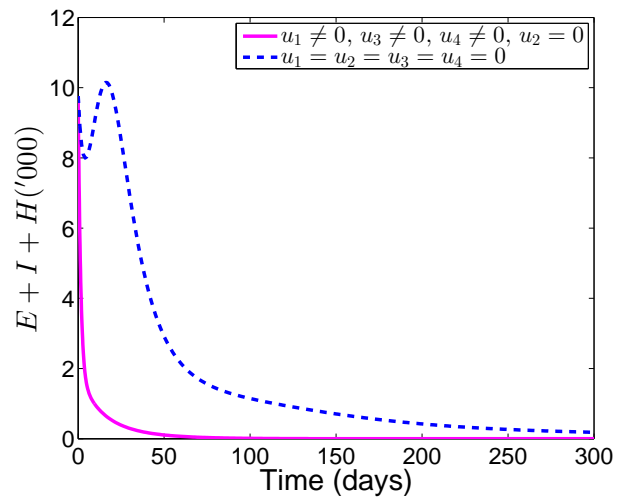

(a) Disease prevalence with and without control Strategy 3

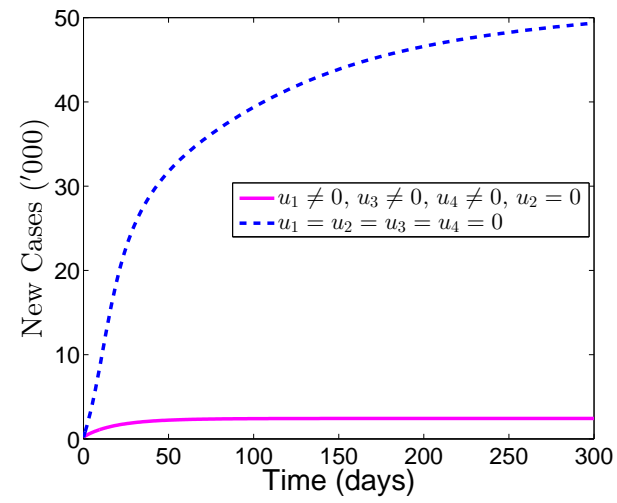

(b) COVID-19 incidence with and without Strategy 3

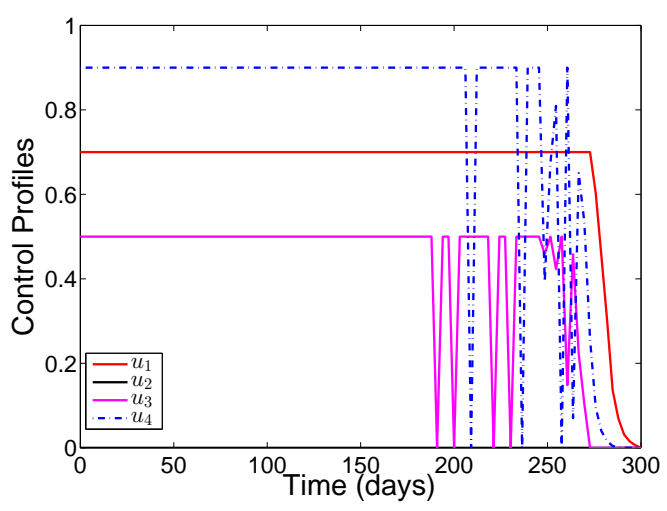

(c) Profiles of optimal controls $u_{1}, u_{3}, u_{4}$ with $u_{2}=0$

Figure 3: Simulations depicting the optimal use of $u_{1}, u_{3}$ and $u_{4}$ only

In Figure 3, the effect of personal protection control $\left(u_{1}(t)\right)$ combined with the efforts of detection control for symptomatic infected individuals $\left(u_{3}(t)\right)$ and management control for hospitalized individuals $\left(u_{4}(t)\right)$ on the dynamics of community spread of COVID-19 is demonstrated. Figure 3 a depicts that the size of COVID-19 prevalence with control intervention diminishes 
more rapidly than when no control is put in place. As shown in Figure 3b, the number of new cases of COVID-19 sharply increased till the end of the disease outbreak, but the number of new cases is successfully managed near the initial value throughout the intervention period. The control profiles in Figure $3 \mathrm{c}$ suggest that personal protection $\left(u_{1}(t)\right.$ is at maximum coverage for about 280 days, counting from the commencement of intervention, before dropping gradually to the lower bound at the final time of control implementation, while the detection control for symptomatic infected individuals $\left(u_{3}(t)\right)$ and the management control for hospitalized humans are at maximum coverage for the first 180 and 205 days, respectively, before decreasing in a stepwise fluctuation manner to the lower bound at the final time.

\subsubsection{Strategy 4-Optimal Use of Personal Protection, Contact Tracing and Testing Control for Asymptomatically In- fected Individuals, Detection Control for Symptomatic Infected Individuals, and Management Control for Hos- pitalized Individuals $\left(u_{1}(t) \neq 0, u_{2}(t) \neq 0, u_{3}(t) \neq 0\right.$, $\left.u_{4}(t) \neq 0\right)$}

Figure 4 illustrates the transmission dynamics of COVID19 with implementation of control strategy that combines personal protection $\left(u_{1}(t)\right)$, contact tracing and testing control for asymptomatic infected individuals $\left(u_{2}(t)\right)$, detection control for symptomatic infected individuals $\left(u_{3}(t)\right)$ and management control for hospitalized individuals $\left(u_{4}(t)\right)$ and without any intervention. As shown in Figure 4a, the disease prevalence with control strategy diminishes more rapidly than when no control is in use. It is also observed from Figure $4 b$ that the number of new cases of COVID-19 is consistently managed close to zero throughout the control implementation period, whereas a rapid increase in the number of new cases is observed when no control strategy is implemented. To obtain this optimal solution, Figure $4 \mathrm{c}$ shows that the four controls are maximally held at the upper bounds ( $70 \%$ for $u_{1}, 50 \%$ for $u_{2}, 50 \%$ for $u_{3}$ and $90 \%$ for $\left.u_{4}\right)$ and minimally held at lower bounds ( $0 \%$ for all the controls) in a stepwise manner over the control intervention period.

\subsection{Efficiency Analysis}

Based on the time series plots in Figs. 1 to 4, it is observed that the implementation of each of the four strategies has similar effect on the dynamics of COVID-19 prevalence and new cases. Thus, it is pertinent to compare the efficacy of each of the four control strategies in reducing the spread of COVID-19 in the population. Therefore, we need to find out the most effective control strategy among the four strategies analysed in this work. In order to identify the most effective strategy among the proposed ones, efficiency analysis is carried out on the four strategies under consideration. Taking a cue from previous studies $[3,43]$, efficiency index, denoted as $\mathbb{E} \mathbb{I}$, is defined as

$$
\mathbb{E} \mathbb{I}=\left(1-\frac{W_{s}}{W_{o}}\right) \times 100 .
$$

In the formula given by (13), $W_{s}$ and $W_{0}$ are the respective cumulative numbers of infected humans with and without control strategy, respectively, which are determined from $\int_{0}^{t_{f}}(E(t)+$

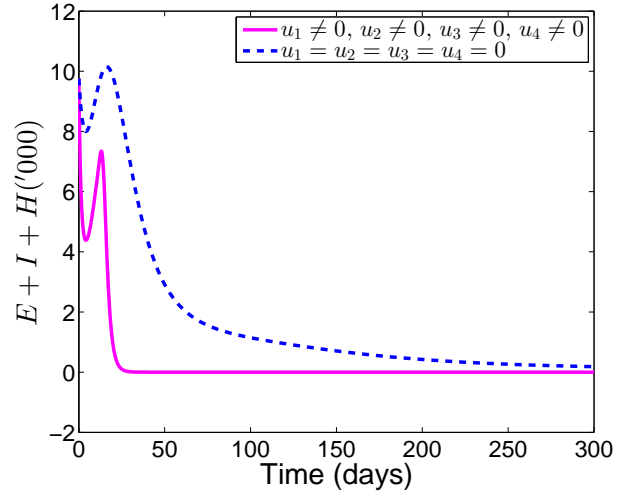

(a) Disease prevalence with and without control Strategy 4

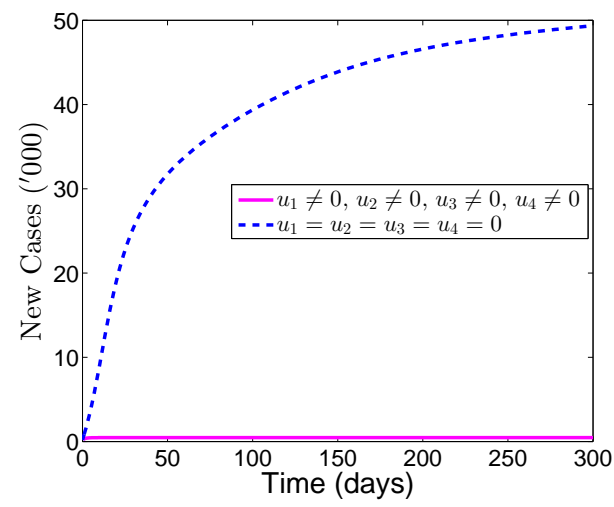

(b) COVID-19 incidence with and without Strategy 4

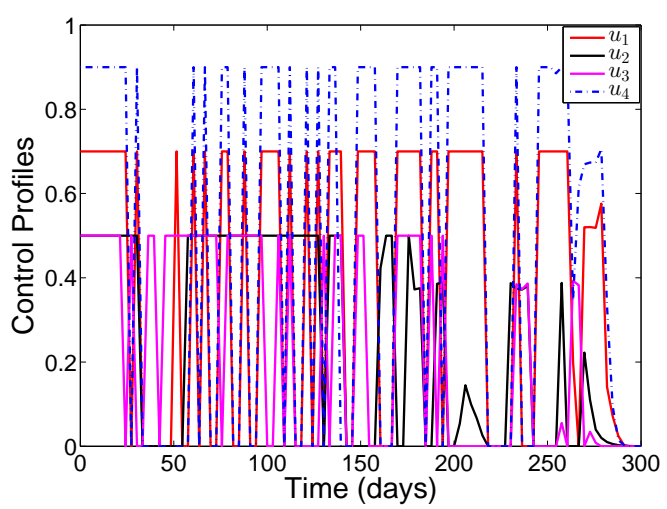

(c) Profiles of optimal controls $u_{1}, u_{2}, u_{3}, u_{4}$

Figure 4: Simulations depicting the optimal use of $u_{1}, u_{2}, u_{3}$ and $u_{4}$

$I(t)+H(t)) d t$, where $t_{f}=300$ days. The strategy with highest efficiency index value is the best strategy $[3,43]$. The value $W_{o}$ is obtained in MATLAB as $W_{o}=\int_{0}^{t_{f}}(E(t)+I(t)+H(t)) d t=$ 550.55 per thousands. The values of $W_{s}$ and the corresponding efficiency index for the four control strategies ranked from lowest to the highest efficiency index are presented in Table 2.

A look at Table 2 shows that Strategy 4 has the highest $\mathbb{E I}$ value, followed by Strategy 2, Strategy 3, and lastly Strategy 1. This result of control strategies ranking in terms of their $\mathbb{E I}$ values suggest that Strategy 4 is the most effective strategy, 
Table 2: Efficiency indices for the four intervention strategies

\begin{tabular}{lcl}
\hline \hline Strategy & $W_{s}\left({ }^{\prime} 000\right)$ & $\mathbb{E I}(\%)$ \\
\hline \hline Strategy 1 & 245.068 & 55.48 \\
Strategy 3 & 233.543 & 57.58 \\
Strategy 2 & 198.493 & 63.94 \\
Strategy 4 & 175.288 & 68.16 \\
\hline \hline
\end{tabular}

followed by Strategy 2, then Strategy 3, while Strategy 1 is the least effective strategy in decreasing the COVID-19 prevalence and the number of new cases in the community.

\section{Conclusion}

In this paper, a mathematical analysis of the novel coronavirus 2019 (COVID-19) pandemic transmission dynamics is carried out to gain insights into the transmission and effective control of the disease using precautionary and control measures. A non-linear mathematical model is formulated by stratifying the total human population into susceptible, exposed (infected but not yet infectious), symptomatic infectious, hospitalized and recovered classes. The model incorporates four timedependent control variables that account for personal protection, contact tracing and testing control for asymptomatic infected humans, detection control for symptomatic infectious humans, and management control for hospitalized humans to examine the impacts of their various combination strategies in minimizing COVID-19 burden in the community. In particular, the developed model is parametrized to mimic the reported cases of COVID-19 in Nigeria. Theoretical analysis of the model is carried out using Pontryagin's maximum principle. The associated optimality system is simulated to examine the effect of the four different proposed strategies on the dynamics of the disease spread. The four strategies are: Strategy 1 (optimal use of personal protection, contact tracing and testing control of asymptomatically infected individuals, and detection control of symptomatic infected individuals), Strategy 2 (optimal use of personal protection, contact tracing and testing control of asymptomatically infected individuals, and management control for hospitalized individuals), Strategy 3 (optimal use of personal protection, detection control of symptomatic infected individuals, and management control for hospitalized individuals) and Strategy 4 (optimal use of personal protection, contact tracing and testing control of asymptomatically infected individuals, detection control of symptomatic infected individuals, and management control for hospitalized individuals). The results reveal that both the prevalence and incidence of the disease can be reduced significantly by implementing any of the four proposed control strategies. Efficiency analysis is carried out to determine the most effective strategy to reduce the community spread of COVID-19 among the four strategies. The results obtained from the analysis further suggests that simultaneous implementation of the optimal use of personal protection, contact tracing and testing control of asymptomatically infected individuals, detection control of symptomatic infected individuals, and management control for hospitalized individuals is the most effective strategy. The epidemiological insight from the result is that to effectively control the dynamics of transmission and spread of COVID-19 in Nigeria (as well as in other countries affected by the pandemic), the implementation of these control and preventive measures should be sustained continuously for an appreciable length of time and relaxed gradually as new cases of the disease become rare and the prevalence rapidly wanes out in the population.

\section{Acknowledgments}

The authors are grateful to the editors and the anonymous reviewers for their constructive comments and suggestions.

\section{References}

[1] M. A. Shereen, S. Khan, A. Kazmi, N. Bashir \& R. Siddique, "COVID19 infection: origin, transmission, and characteristics of human coronaviruses", Journal of Advanced Research, 24 (2020) 91.

[2] D. Okuonghae \& A. Omame "Analysis of a mathematical model for COVID-19 population dynamics in Lagos, Nigeria”, Chaos, Solitons and Fractals, 139 (2020) 110032.

[3] A. Abidemi, Z. M. Zainuddin \& N. A. B. Aziz, "Impact of control interventions on COVID-19 population dynamics in Malaysia: a mathematical study", The European Physical Journal Plus, 136 (2021) 237.

[4] R. Wu, L. Wang, H. C. D. Kuo, A. Shannar, R. Peter, P. J. Chou, S. Li, R. Hudlikar, X. Liu, Z. Liu, G. J. Poiani, L. Amorosa, L. Brunetti \& A. N. Kong, "An update on current therapeutic drugs treating COVID-19", Current Pharmacology Reports, 6 (2020) 56.

[5] F. Galluccio, T. Ergonenc, A. G. Martos, A. E. Allam, M. Pérez-Herrero, R. Aguilar, G. Emmi, M. Spinicci, I. T. Juan \& M. Fajardo-Pérez, "Treatment algorithm for COVID-19: a multidisciplinary point of view, Clinical Rheumatology, 39 (2020) 2077.

[6] R. C. Becker, "COVID-19 treatment update: follow the scientific evidence", Journal of Thrombosis and Thrombolysis, 50 (2020) 43.

[7] C. J. Galvin, Y. C. Li, S. Malwade \& S. Syed-Abdul, "COVID-19 preventive measures showing an unintended decline in infectious diseases in Taiwan", International Journal of Infectious Diseases, 98 (2020) 18.

[8] N. H. Shah, A. H. Suthar \& E. N. Jayswal, "Control strategies to curtail transmission of COVID-19", International Journal of Mathematics and Mathematical Sciences, 2020 (2020).

[9] C. Tsay, F. Lejarza, M. A. Stadtherr \& M. Baldea, "Modeling, state estimation, and optimal control for the US COVIDâ19 outbreak", Scientific Reports, 10 (2020) 10711.

[10] C. N. Ngonghala, E. Iboi, S. Eikenberry, M. Scotch, C. R. MacIntyre, M. H. Bonds \& A. B. Gumel, "Mathematical assessment of the impact of non-pharmaceutical interventions on curtailing the 2019 novel coronavirus", Mathematical Biosciences, 325 (2020) 108364.

[11] World Health Organization, "Coronavirus (COVID-19) Dashboard" (2021) Accessed on 5 December, 2021. https://covid19.who.int/.

[12] S. Olaniyi, O. S. Obabiyi, K. O. Okosun, A. T. Oladipo \& S. O. Adewale, "Mathematical modelling and optimal cost-effective control of COVID19 transmission dynamics", The European Physical Journal Plus, 135 (2020) 938.

[13] Nigeria Centre for Disease Control, "COVID-19 Outbreak in Nigeria situation report; NCDC: Abuja, Nigeria", Accessed on 8th August, 2020. https://ncdc.gov.ng/diseases/sitreps.

[14] Nigeria Centre for Disease Control, "COVID-19 Nigeria" (2021). https://covid19.ncdc.gov.ng/report/. Accessed on 5 December, 2021.

[15] C. Ohia, A. S. Bakarey \& T. Ahmad, "COVID-19 and Nigeria: putting the realities in context", International Journal of Infectious Diseases, 95 (2020) 279.

[16] B. Ebenso \& A. Otu, "Can Nigeria contain the COVID-19 outbreak using lessons from recent epidemics?", Lancet Global Health, 8 (2020) e770.

[17] I. A. Osseni, "COVID-19 pandemic in Sub-Saharan Africa: preparedness, response, and hidden potentials", Tropical Medicine and Health, 48 (2020) 48. 
[18] C. O. Ijalana \& T. T. Yusuf, "Optimal control strategy for Hepatitis B virus epidemic in areas of high endemicity", International Journal of Scientific and Innovative Mathematical Research, 15 (2017) 28.

[19] T. T. Yusuf \& A. O. Olayinka, "Optimal control of Meningococcal Meningitis transmission dynamics: A case study of Nigeria", IOSR Journal of Mathematics, 15 (2019) 13.

[20] L. L. Obsu \& S. F. Balcha, "Optimal control strategies for the transmission risk of COVID-19", Journal of Biological Dynamics, 1 (2020) 590.

[21] A. Yousefpour, H. Jahanshahi \& S. Bekiros, "Optimal policies for control of the novel coronavirus disease (COVID-19) outbreak", Chaos, Solitons and Fractals, 136 (2020) 109883.

[22] A. I. Abioye, O. J. Peter, H. A. Ogunseye, F. A. Oguntolu, K. Oshinubi, A. A. Ibrahim \& I. Khan, "Mathematical model of COVID-19 in Nigeria with optimal control", Results in Physics, 28 (2021) 104598.

[23] A. I. Abioye, M. D. Umoh, O. J. Peter, H. O. Edogbanya, F. A. Oguntolu, O. Kayode \& S. Amadiegwu, "Forecasting of COVID-19 pandemic in Nigeria using real statistical data", Communications in Mathematical Biology and Neuroscience, (2021) 1.

[24] M. L. Diagne, H. Rwezaura, S. Y. Tchoumi \& J. M. Tchuenche, "A mathematical model of COVID-19 with vaccination and treatment", Computational and Mathematical Methods in Medicine, 1250129 (2021) 1.

[25] S. S. Musa, S. Qureshi, S. Zhao, A. Yusuf, U. T. Mustapha \& D. He, "Mathematical modeling of COVID-19 epidemic with effect of awareness programs", Infectious Disease Modelling, 6 (2021) 448.

[26] O. J. Peter, A. S. Shaikh, M. O. Ibrahim, K. S. Nisar, D. Baleanu, I. Khan \& A. I. Abioye, "Analysis and dynamics of fractional order mathematical model of COVID-19 in Nigeria using Atangana-Baleanu operator", Computers, Materials and Continua, (2021) 1823.

[27] O. J. Peter, S. Qureshi, A. Yusuf, M. Al-Shomrani \& A. A. Idowu, "A new mathematical model of COVID-19 using real data from Pakistan", Results in Physics, 24 (2021) 104098.

[28] T. T. Yusuf, A. S. Afolabi, E. J. Dansu \& A. Abidemi, "Modelling the novel coronavirus (COVID-19) transmission dynamics with qualitative analysis: a case study of Nigeria", Submitted Manuscript.

[29] A. Abidemi, R. Ahmad \& N. A. B. Aziz, "Global stability and optimal control of dengue with two coexisting virus serotypes", MATEMATIKA: Malaysian Journal of Industrial and Applied Mathematics, 35 (2019) 149.

[30] A. Abidemi \& N. A. B. Aziz, "Optimal control strategies for dengue fever spread in Johor, Malaysia", Computer Methods and Programs in
Biomedicine, 196 (2020) 105585.

[31] J. K. K. Asamoah, M. A. Owusu, Z. Jin, F. T. Oduro, A. Abidemi \& E. O. Gyasi, "Global stability and cost-effectiveness analysis of COVID19 considering the impact of the environment: using data from Ghana", Chaos Solitons and Fractals, 140 (2020) 110103

[32] T. T. Yusuf \& F. Benyah, "Optimal control of vaccination and treatment for an SIR epidemiological model", World Journal of Modelling and Simulation, 8 (2012) 194.

[33] T. T. Yusuf \& F. Benyah, "Optimal strategy for controlling the spread of HIV/AIDS disease: A case study of South Africa", Journal of Biological Dynamics, 6 (2012) 475.

[34] W. H. Fleming \& R. W. Rishel. Deterministic and stochastic optimal control. Springer-Verlag, New York, (1975).

[35] E. A Coddington \& N. Levinson. Theory of ordinary differential equations. McGraw Hill, New York, (1955).

[36] L. S. Pontryagin, V. G. Boltyanskii, R. V. Gamkrelidze \& E. F. Mishchenko. The mathematical theory of optimal processes. Gordon and Breach Science Publishers, (1986).

[37] R. F. Hartl, "Optimal control of non-linear advertising models with replenishable budget", Optimal Control Applications and Methods, 3 (1982) 53.

[38] United Nation, "World population prospects", Accessed on 20th August, 2020. https://esa.un.org/unpd/wpp/DataQuery/.

[39] T. T. Yusuf, "Mathematical modelling and simulation of Meningoccal Meningitis transmission dynamics", FUTA Journal of Research in Science, 14 (2018) 94.

[40] World Health Organization, "Coronavirus Disease (COVID2019) Situation Reports", (2020) Accessed on 23 May, 2020. https://www.who.int/emergencies/diseases/novel-coronavirus2019/situation-reports.

[41] Nigeria Centre for Disease Control, "COVID-19 Outbreak in Nigeria Situation Report", NCDC: Abuja, Nigeria (2020). Accessed on 23 May, 2020.

[42] S. Lenhart \& J. T. Workman. Optimal control applied to biological models. Taylor \& Francis, Boca Raton, FL, (2007).

[43] S. A. Carvalho, S. O. da Silva \& I. da Cunha Charret, "Mathematical modeling of dengue epidemic: control methods and vaccination strategies", Theory in Biosciences, 138 (2019) 223. 